\title{
End Systolic Volume Imaging
}

National Cancer Institute

\section{Source}

National Cancer Institute. End Systolic Volume Imaging. NCI Thesaurus. Code C94894.

Imaged ventricular blood pool capacity within the endocardial surface in a gated scan during the time of systole. 